Die Autoren der folgenden Beiträge setzen sich kritisch mit inhaltlichen und formalen Aspekten des Tribünen-Artikels von G. Kocher zum Thema «Fortschritte in der Medizin» auseinander, der am 6. September 2006 in der SÄZ Nr. 36 erschienen ist.

Die Redaktion

\title{
Innovationen ausserhalb der ... fachmedizinischen Produktinnovationen
}

Reto Tscholl

1 Kocher G. Fortschritte in der Medizin - ja, aber ... Schweiz Ärztezeitung. 2006;87(36):1555-9.

Korrespondenz:

Dr. med. Reto Tscholl

Buchweg 10

CH-5018 Erlinsbach
Unter dem Titel «Fortschritte in der Medizin - ja, aber ...» zählt G. Kocher in seiner Tabelle 1 die Probleme des Gesundheitswesens umfassend auf. Diese werden einmal mehr treffend diagnostiziert.

Demgegenüber erweisen sich die Therapievorschläge, wie Pflegeforschung, Kontrolle der Fortbildung, Statistiken, Förderung von Patientenverbänden, Planung der Gesundheitsversorgung u.a.m., die in seiner Tabelle 2 aufgelistet sind, als gutgemeinte Fernziele, die aber keine unmittelbare Wirkung auf die Kosten ausüben werden. Leider wird die Forderung nach der «Schaffung richtiger Anreize», die für die Eindämmung der steigenden Kosten entscheidend wäre, nicht so detailliert ausgeführt, dass sie umsetzbar wäre. An dieser Stelle soll deshalb versucht werden, die Schaffung dieser richtigen Anreize zu konkretisieren.

Die zwei Hauptursachen der Kostensteigerung im Gesundheitswesen, der medizinische Fortschritt und die Demographie, sind nicht eliminierbar. Eine steigende Nachfrage erzeugt bei politisch fixierten Preisen - also bei Tarifen zwangsläufig steigende Kosten. Wenn in der ganzen Wirtschaft mangelnder Wettbewerb zu Preisanstiegen und umgekehrt Wettbewerb zu tieferen Preisen führt, ist nicht einzusehen, wieso der Wettbewerb nicht auch im Gesundheitssektor zu sinkenden Preisen führen sollte.

Im Gesundheitssektor existiert aber bisher kein Markt, in dem sich Marktpreise unter Konkurrenz bilden könnten, sondern eine Planwirtschaft mit Tarifen. Innerhalb der Planwirtschaft scheiterten bisher weltweit alle Versuche, die Gesundheitskosten zu senken. Wenn die Kostensenkung überhaupt noch ein Ziel ist, muss die
Planwirtschaft durch einen Markt ersetzt werden, der drei banale Kennzeichen haben muss: 1. Preiskonkurrenz unter den Anbietern; 2. Kaufentscheid durch den Zahler; 3. finanzielle Konsequenz für den Käufer.

\section{Preiskonkurrenz unter den Ärzten bzw. den Spitälern}

Wie in jedem Markt müssen die Anbieter die Preise für ihre medizinischen Dienstleistungen festlegen, sie publizieren und der Konkurrenzlage anpassen.

Die Preise beziehen sich auf definierte Leistungspakete (DRG). Für zeitlich absehbare Leistungen (z.B. Operationen) existieren DRGs bereits. Bei zeitlich nicht absehbaren Leistungen für chronisch Kranke würden die Preise für die Betreuung gemäss einem Servicevertrag erhoben, ähnlich wie für die Wartung von technischen Anlagen. Für notfallmässig erbrachte Leistungen könnte jener Preis gefordert werden, der am Ereignistag für die gefragte Leistung am Markt durchschnittlich galt.

Die DRGs, auch die künftigen Swiss-DRGs, dürfen nicht als blosse Tarifpakete nur gerade zur Erleichterung der Rechnungsstellung, sondern als Offerten mit Preisangabe verwendet werden.

\section{Der Patient ist Zahler und fällt den Kaufentscheid}

Kunde im Gesundheitswesen ist der Patient, weil er die Gelder über Steuern und Prämien aufbringt, nicht die Krankenkasse, die Gelder nur weiterleitet. Der Patient ist fähig, über seine Behandlung zu entscheiden, wenn er über sein eigenes medizinisches Problem und die Qualität der offerierten Leistungen informiert ist. 
An Informationen braucht er nur jene, die sein Problem betreffen und die ihm sein Hausarzt ohnehin geben muss. Zusätzlich hat er über Zweitmeinungen Zugang zum ganzen Wissen medizinischer Zentren, soweit es ihn betrifft, mit welchem die Vertrauensärzte der Versicherer nie gleichziehen können, weil sie nicht klinisch tätig sind. Der Patient ist zudem motivierter, dieses Wissen zu erwerben und zu nutzen, als es Versicherungsärzte je sein können.

Die Resultatqualität (Outcomequalität) medizinischer Leistungen zu messen, ist aufwendig. Diese Art der Qualität wurde bisher meist nur in Studien, aber nicht im Rahmen der normalen medizinischen Versorgung verwendet. Vor kurzem wurde sie aber in einem «bürgerlichen Modellvorschlag» gefordert (NZZ, 30. August 2006). Jedenfalls ist für die Zukunft die Garantie einer Basisqualität anzustreben, unabhängig davon, ob weiter Tarife gelten wie jetzt oder ob man auf Marktpreise umstellt.

\section{Die Kostenbeteiligung des Patienten}

Wenn der Preis den Kaufentscheid des Patienten beeinflussen soll, muss dieser die finanziellen Konsequenzen seiner Wahl spüren. Der Patient bezahlt innerhalb eines Selbstbehalts einen Kostenanteil, der dem Preis der konsumierten Leistung proportional ist. Der Selbstbehalt wird in der Grundversicherung abhängig vom Einkommen festgelegt. In der Zusatzversicherung kann er frei gewählt werden.

Der Selbstbehalt gewährleistet, dass der Patient ein persönliches finanzielles Interesse daran hat, seine Behandlung wie auch den Leistungserbringer möglichst preisgünstig zu wählen. Dadurch sind wiederum die Anbieter gezwungen, ihre Preise so tief anzusetzen, dass der Patient nicht zu einem fachlich gleich angesehenen, aber günstigeren Konkurrenten abwandert.

Der in dieser Weise einkommensabhängige Preis hat gegenüber einer einkommensabhängigen Prämie den grossen Vorteil, dass der Patient innerhalb des Selbstbehalts den im Schadenfall geschuldeten Betrag bestimmen kann, indem er die durch seine Wahl entstehenden Kosten und damit seinen Kostenanteil steuert, wogegen er keinen Einfluss auf eine einkommensabhängige Prämie hat und diese wiederum keinerlei Wirkung auf die Kosten ausüben kann.

\section{Wirkung der Preiskonkurrenz im Gesundheitswesen}

Der Preiskampf wird die Anbieter zwingen, Unnötiges innerhalb der offerierten DRGs wegzulassen und Wiederholungen (Röntgen, Labor) zu vermeiden. Unter dem Preisdruck werden auch die bürokratischen Ungetüme weitgehend abgebaut werden, die entstanden sind, weil staatliche Strukturen (Gesundheitsdirektion, Spitaldirektion, Spitalverwaltungen) und privatwirtschaftliche Organisationsformen (VR, CEO) einfach übereinandergeschichtet wurden. Sie sind teuer und behindern jene, die am Patienten die Leistungen eigentlich erbringen. Das Personal, das für die Verwaltung und das Management tatsächlich nötig ist, soll nicht in praxisfernen Stäben (Gesundheitsdirektionen, Spitalverwaltungen) aufgebläht werden, sondern muss zahlenmässig reduziert am «point of sale», also nahe an oder in den Kliniken bzw. Instituten, eingesetzt werden.

\section{Konklusion}

Nur die Preiskonkurrenz erzeugt einen Zwang, günstige Preise zu offerieren, was schliesslich die Kosten auch im Gesundheitssektor senken wird. Dieser Zwang fehlt bei Tarifen. Daran würde auch die Vertragsfreiheit nichts ändern. Sie ist zwar eine Voraussetzung, dass die Preiskonkurrenz im Gesundheitssektor stattfinden kann, aber als isolierte Massnahme spart sie keine Kosten. Den Kontrahierungszwang aufzuheben, ohne gleichzeitig den Wettbewerb im Gesundheitswesen einzuführen, wäre so sinnvoll, wie einem Auto im Leergang Vollgas zu geben und nicht abzufahren.

Auch wenn die Preiskonkurrenz im Gesundheitswesen bisher nie praktiziert wurde, ist plausibel, dass sie die Gesundheitskosten senken wird, weil sich, ökonomisch gesehen, medizinische von andern Dienstleistungen nicht unterscheiden. Genau so wie die Preiskonkurrenz zwischen Grossverteilern oder zwischen Telekommunikationsanbietern die Preise günstig beeinflusst, wird der Preiskampf auch im Gesundheitswesen verhindern, dass die Kosten ungebremst weitersteigen.

Ob die Ärzteschaft wagen wird, sich einer Preiskonkurrenz auszusetzen, ist vorläufig ungewiss. 
Kurt Bösch

Der Begriff «early adopter» (englisch für: frühzeitiger Anwender) bezeichnet einen Menschen, der die neusten technischen Errungenschaften oder die neusten Varianten von Produkten erwirbt, obwohl diese sehr teuer und oftmals unausgereift sind. Meistens werden Produkte für «early adopter» noch vor der Fertigstellung eines entsprechenden Standards vertrieben, womit sie häufig innerhalb kurzer Zeit praktisch wertlos werden.

Korrespondenz:

Dr. med. Kurt Bösch

Schlossbach

Postfach

CH-9404 Rorschacherberg
Sie sind, wie Sie wissen, liebe Kolleginnen und Kollegen, alle Leistungserbringer, einige unter Ihnen sind gar Adopter? Diese Unwörter, die man für uns bereithält, tönen noch scheusslicher, wenn man sie «feminisiert», die Leistungserbringerin oder die Adopterin. Beide stehen noch nicht in meinem Duden, 22. Auflage, 2000 (wenn man diesen bereits veralteten Duden zurückbringt, bekommt man übrigens 7 Franken 40 gutgeschrieben!). Die neuste, 25. Auflage werde ich dann nach der übernächsten Schreibreform erwerben, dann, wenn es vielleicht heissen wird «zu Recht weisen» statt «zurechtweisen».

Dem Autor des Artikels «Fortschritte in der Medizin - ja, aber ...» in der SÄZ Nr. 36 ist es gelungen, den Adopter in mein Sprachbewusstsein $\mathrm{zu}$ bringen, der Leistungserbringer war schon drin. Was Wikipedia über ihn, den (eigentlich early) Adopter*, zu sagen weiss, ist aufschlussreich, erheiternd und wenig schmeichelhaft zugleich. Die von mir konsultierten Wörterbücher wissen zurzeit noch nichts von ihm, auch nichts vom Leistungserbringer, dessen Herkunft schleierhaft ist. Der letztere treibt sich seit weniger als sechs Jahren in der Gesundheits- und Kassenpolitik, aber noch nicht in den gängigen Nachschlagewerken herum.

Sei dem wie ihm sei, den Leser stören diese Neologismen vielleicht nicht und er geht, wie ich selbst, im grossen ganzen mit dem Autor des genannten Artikels einig (so er ihn gelesen hat): In der Medizin gibt es Fortschritte, und die Fortschritte kosten etwas, es braucht aber noch mehr Fortschritte und die werden auch kosten. Man müsste Wege finden, die Kosten in Grenzen zu halten. Der Artikel beschäftigt sich vor allem mit den Unzulänglichkeiten im Gesundheitswesen, mit Kostenfolgen und etwas weniges mit anderen Konsequenzen des Fortschrittes (auch des fehlenden), wie z.B. die notwendige breitflächige und rasche Anwendung bzw. Umsetzung neuer Erkenntnisse oder Möglichkeiten (eben z. B. durch die Adopter).

Der Autor präsentiert in Tabellenform eine Aufstellung der für ihn augenscheinlichen Mängel und Schwächen des Gesundheitssystems. Die Tabelle ist kunterbunt, weder nach Prioritäten gruppiert, noch irgendwie gewichtet, aber immerhin alphabetisch geordnet. Die kaum reflektierte Aufzählung der Mängel und Schwächen kann fast beleidigend empfunden werden, auch wenn man sie zum Teil der fehlenden Zuständigkeit des Ökonomen zuschreiben darf. Man ist geneigt, bei jeder der 25 Positionen der Tabelle (die Sie, liebe Kollegen, leider sicher nicht mehr zur Hand haben) zu fragen, wie der Autor das nun meint, was er sich geändert wünscht, wieweit er Änderungen für möglich hält, was konkret er sich von den Änderungen verspricht. Die Aufstellung ist aus der Sicht eines Ökonomen zusammengestiefelt, der in dieser Tabelle dem Gesundheitswesen auch «ungeklärte Ziele» und «Unterversorgung in anderen (?) Gebieten» vorwirft. Aber er weiss offenbar auch um die Mängel in der Bodenrettung, er weiss von dem Zuviel an Leistung und moniert den Zuständigkeitswirrwarr. Er führt auch den ungenügenden Kampf gegen Tabak ins Feld, verliert aber über Alkohol und Übergewicht kein Wort (es sei denn, die gehörten zum Kapitel unterentwickelte Präventivmedizin), er weiss auch von unterentwickelter Palliativmedizin.

Müssen Sie, liebe aktive Kollegen, sich das gefallen lassen? Man könnte empfindlich reagieren auf diesen Beitrag und ihn als Rundumbeschuldigung der säumigen Involvierten und Mitverantwortlichen interpretieren. Als das war er sicher nicht gemeint.

Eine zweite Tabelle zählt Beispiele für die «Fortschritte und Innovationen ausserhalb der traditionellen fachmedizinischen Produktinnovationen» auf. Sie werden im Text allerdings als Reformvorhaben, die nur mit ungeheurem Aufwand an Streit und Zeit realisiert werden können - wenn überhaupt -, beschrieben. Die Tabelle ist nicht mehr alphabetisch geführt und vermischt Erreichtes mit zusätzlich Notwendigem und mit Gewünschtem. Im Text wird darauf hingewiesen, dass eklatante Forschungslücken bestehen im Krankenhausmanagement, in der Pflegewissenschaft, in der Allgemeinmedizin, Ethik, Medizinsoziologie, Versicherungsmedizin, eigentlich überall, wieder eine Rundumbeschuldigung.

Wir meinen, der oben fragmentarisch besprochene Beitrag sei kein Beitrag zu irgendwelchen Lösungen. Si tacuisses...

PS: Der Autor der oben besprochenen Studie publizierte vor einigen Monaten auch die OECDZahlen über die Gesundheitskosten. In einem Leserbrief bedeutete ich, dass wir die Zahlen selber lesen könnten, dass aber eine Erklärung für die fast unglaubwürdigen Werte gesucht werden 
müsste. Der Autor ging damals nicht auf meinen Leserbrief ein und erwähnt meinen Einwand auch in seiner neusten Publikation in der SÄZ Nr. 38, «Schweiz wieder im zweiten und dritten Rang», nicht und präsentiert wiederum Zahlenreihenfolgen aus einer CD der OECD, die er sich für $€ 80$ (früherer Preis, erwähnt er, $€$ 200) erworben hat. Er versucht in keiner Weise, die grossen Differenzen und die eigenartigen Rangfolgen $\mathrm{zu}$ erklären und $\mathrm{zu}$ analysieren und vielleicht Lehren aufzuzeigen, die daraus gefolgert werden könnten. Er ist offenbar auch überzeugt, dass die BIP-Werte der 25 Länder ohne Zögern verglichen werden können (weil sie von der sakrosankten OECD stammen) und dass die OECD tatsächlich sogar weiss, wie viele CT, MIR im Jahre 2004 z. B. in Mexiko, in Südkorea und in Griechenland vorhanden waren. Die Idee, dass vielleicht die Vergleichbarkeit der Zahlen in Frage gestellt werden könnte, kommt nicht auf. Anschliessend schreibt er: «[...] es gibt Länder mit anerkannt guten Gesundheitssystemen, die pro Kopf nur die Hälfte oder noch weniger ausgeben als Länder mit vergleichbaren Gesundheitssystemen». Er entdeckt dann, «dass die Qualität der Gesundheitsversorgung von zahlreichen Faktoren abhängt und dass die Höhe der Gesundheitskosten nur einer von vielen und nicht der entscheidende Faktor sei.» Tableau!

PPS. In den letzten Tagen und Wochen haben wir aus den Zeitungen erfahren können, dass die anonyme WHO und die ebenso anonyme OECD uns nicht nur das zweitteuerste Gesundheitswesen attributiert, sondern dieses gleich noch der Ineffizienz und des Ungenügens bezichtigt. Vor diesem Hintergrund gewinnt der obige Artikel an Bedeutung. Die Frage nach den Urhebern der Behauptung, nach dem Wahrheitsgehalt und der Objektivität, nach den Vergleichskriterien, nach den Ursachen, nach den Bewältigungsmöglichkeiten ist vordringlich. Werden wir in dieser Zeitung darüber lesen können? Wer gibt uns Auskunft, aber bitte kompetent? 